\title{
Conversões entre as representações em linguagem natural e algébrica de função quadrática: uma análise do fenômeno de congruência semântica e equivalência referencial
}

\author{
Andreza Santana da Silva \\ (iD \\ Universidade Federal de \\ Pernambuco
}

\andrezass19@hotmail.com

Rosinalda Aurora de Melo

Teles (iD

Universidade Federal de

Pernambuco

פrosinaldateles@yahoo.com.br

\author{
Conversions between representations in natural and algebraic \\ language with quadratic function: an analysis of the phenomenon \\ of semantic congruence and referential equivalence
}

\begin{abstract}
This article, part of a master's study in Mathematical and Technological Education, aims to analyze the phenomenon of semantic congruence and referential equivalence in activities of converting the record in natural language to the algebraic record in a quadratic function presented in a textbook of the 1st series of the high school. The Theory of Semiotic Representation Records was the theoretical contribution and the methodological perspective for the analysis. Only the chapter of the textbook that dealt with quadratic function was analyzed, containing 67 questions with a total of 158 items, among which 46 were categorized as conversion from natural to algebraic language, subdivided when they needed an auxiliary representation record. The analysis was based on the three criteria established by Duval [2] for the variation of semantic congruence and noncongruence: Semantic correspondence of the significant elements, terminal semantic univocity and order within the organization of the units and the referential equivalence. Items that kept all the criteria were considered as congruent, and those that did not keep one, two or three criteria were considered as non-congruent, being, respectively, low, medium and high degree of semantic non-congruence. In general, it can be seen that in this type of conversion there is a higher prevalence in activities of low and medium degree of semantic non-congruence.
\end{abstract}

Key words: Phenomenon of congruence and semantic non-congruence; Referential equivalence; Conversion; Quadratic function; Textbook.

\begin{abstract}
Resumo
Este artigo, recorte de um estudo de mestrado em Educação Matemática e Tecnológica, objetiva analisar o fenômeno de congruência semântica e equivalência referencial em atividades de conversão do registro em linguagem natural para o registro algébrico em função quadrática apresentadas em um livro didático da $1^{\mathrm{a}}$ série do ensino médio. A Teoria dos Registros de Representação Semiótica foi o aporte teórico e o olhar metodológico para a análise. Foi analisado apenas o capítulo do livro didático que tratava de função quadrática, contendo 67 questões com um total de 158 itens dentre os quais 46 foram categorizados como conversão da representação em linguagem natural para algébrica, subdivididas quando necessitavam de um registro de representação auxiliar. A análise baseou-se nos três critérios estabelecidos por Duval [2] para a variação de congruência e não congruência semântica: Correspondência semântica dos elementos significantes, univocidade semântica terminal e ordem dentro da organização das unidades e a equivalência referencial. Foram considerados como congruentes os itens que conservassem todos os critérios, e não congruentes os que não conservassem um, dois ou três critérios, sendo respectivamente, baixo, médio e alto grau de não congruência semântica. De maneira geral, pode-se perceber que nesse tipo de conversão existem maior prevalência em atividades de baixo e médio grau de não congruência semântica.

Palavras-chave: Fenômeno de congruência e não congruência semântica; Equivalência referencial; Conversão; Função quadrática; Livro didático.
\end{abstract}




\section{INTRODUÇÃO}

Na matemática, uma das atividades que desencadeia dificuldades é transpor um enunciado em língua natural para linguagem matemática, seja numericamente ou algebricamente, pois dependendo do contexto em que as palavras em linguagem natural sejam empregadas, elas podem gerar uma confusão ou até mesmo uma incompreensão por parte dos estudantes.

Existe uma distância cognitiva entre a linguagem natural e outros registros, isso explica a dificuldade em conversões partindo dos enunciados em linguagem natural [3]. Na Teoria dos Registros de Representação Semiótica (TRRS) de Raymond Duval, os registros são "ferramentas que permitem analisar todas as produções matemáticas, e em primeiro lugar aquelas construídas com objetivo de ensino ou de aprendizagem" [3] (p. 104). É importante compreender que só são registros as representações semióticas que obedecem às três funções cognitivas: comunicação, objetivação e tratamento, que correspondem, respectivamente, a "transmissão de uma mensagem ou de uma informação entre indivíduos, requerendo a utilização de um código comum", a segunda "é a função que permite a um sujeito de tomar consciência daquilo que até então não tinha feito. É o trabalho de exteriorização"; e a última é "a função de transformar uma representação em outra, utilizando unicamente as possibilidades de funcionamento do sistema de representação mobilizado" [4] (p. 3).

O que Duval assume como a atividade de conversão, é a transformação que acontece externa ao registro, ou seja, é a passagem de uma representação a outra e que acontecem em registros distintos [2]. Como é o caso da passagem da linguagem natural para algébrica, que dito nos termos empregados na TRRS é a conversão da representação que parte do registro em linguagem natural para o registro algébrico.

Neste texto, recorte do estudo de mestrado, intitulado Registro de Representação Semiótica e Função Quadrática: um olhar sobre o ensino e a abordagem no livro didático $^{1}$, desenvolvido no Programa de Pós-Graduação em Educação Matemática e Tecnológica (EDUMATEC/UFPE), discute-se especificamente a análise do fenômeno de congruência semântica e equivalência referencial em atividades apresentadas em um livro didático da $1^{\mathrm{a}}$ série do ensino médio sobre função quadrática, mas especificamente, com foco nas conversões no sentido registro em linguagem natural (RLN) para o registro algébrico (RA), tendo em vista que este tipo de conversão foi o mais enfatizado.

Essa análise é relevante, pois "a variação de congruência e não congruência se-

\footnotetext{
${ }^{1}$ Um artigo que consta a análise geral da abordagem de função quadrática no livro didático e no ensino e que também foi retirado da dissertação, é intitulado Convergências entre a abordagem do livro didático e o ensino de função quadrática: um olhar sob o ponto de vista dos registros de representação semiótica, e encontra-se em http://revistas.pucsp.br/emp/article/view/47455.
} 
mântica é uma das maiores causas da incompreensão ou erros de interpretação dos enunciados do problema para os alunos" [3] (p. 121). Duval ainda deixa claro que existem em maior número os fenômenos de não congruência do que os fenômenos de congruência, e pela riqueza diversa de registros, "devem ser estudados caso a caso, para cada atividade ou cada problema que propomos" [3] (p. 124).

\section{O FENÔMENO DE CONGRUÊNCIA SEMÂNTICA E A EQUIVALÊNCIA REFERENCIAL}

Duval afirma que uma atividade essencial para o pensamento matemático é "substituir uma formulação ou uma apresentação por outra, referencialmente equivalente" e isso requer algumas condições "para que haja sentido no pensamento natural: a continuidade semântica e a associatividade entre as expressões a serem substituídas" [1] (p. 99). Diante disso, na atividade de conversão, por exemplo, ao converter uma expressão em linguagem natural para a representação em linguagem algébrica, fazse necessária uma substituição por símbolos algébricos e numéricos referencialmente equivalentes e que podem ser semanticamente congruentes ou não.

Uma das maiores dificuldades ao converter partindo do registro em linguagem natural para o registro algébrico, dá-se pela substituição seguindo a ordem em que o enunciado é escrito, sem que seja identificada a equivalência referencial entre os termos [6]. Concomitantemente, Duval expõe que "um dos obstáculos encontrados por muitos alunos na aprendizagem de matemática está ligado ao fato de que a equivalência referencial destaca-se da congruência semântica e, no entanto, o funcionamento espontâneo do pensamento segue prioritariamente a congruência semântica" [1] (p. 101). Por esse motivo, conversões não congruentes são muito mais complexas de serem realizadas pelos alunos.

Assim, para determinar se duas representações são congruentes ou não congruentes, são estabelecidos três critérios [2]:

I. Correspondência semântica dos elementos significantes;

II. Univocidade semântica terminal;

III. Ordem dentro da organização das unidades.

A correspondência semântica dos elementos significantes refere-se à associação de uma unidade significante ${ }^{2}$ de uma representação a uma única unidade significante de outra representação. Sendo assim os termos matematicamente pertinentes devem

\footnotetext{
${ }^{2}$ Nesse estudo, os termos: unidade significante, unidade de sentido e elemento significante são tratados como sinônimos.
} 
ter apenas um símbolo associado a ele quando convertido no registro algébrico. No exemplo 1 a seguir:

(1) Minha idade multiplicada por 3 e subtraída por 15 será igual ao dobro da minha idade mais 6. Qual é a minha idade?

A conversão desse exemplo no registro algébrico corresponde a seguinte equação: $x \cdot 3-15=2 \cdot x+6$. Percebe-se, no entanto, que a palavra dobro está ligada a dois signos: o numeral 2 e o símbolo da multiplicação “.”, dessa forma, o critério (I) não é satisfeito neste exemplo.

Ainda pelo exemplo (1) verifica-se que o critério (III) é respeitado, haja vista que ao fazer a leitura do registro em linguagem natural e em linguagem algébrica, da esquerda para direita, correspondem a mesma ordem na organização das unidades significantes. Para Duval [2] (p. 69) "esse critério é, sobretudo, importante quando se trata de comparar frases e fórmulas literais", assim, não é simplesmente "traduzir" as palavras, na mesma ordem em que estão, para a linguagem algébrica, pois existirão casos que tenderão ao erro se forem pensados dessa forma, como no exemplo 2:

(2) Se subtrairmos um número ao triplo da idade de Joana, o resultado será 27. Qual a idade de Joana, sabendo que o número subtraído foi seis?

Se os alunos pensarem em apenas "traduzir" o que está em palavras para a linguagem algébrica na mesma ordem, chegarão ao equívoco de escreverem a equação $3 \cdot x-y=27-6$, por exemplo. Pensando o número qualquer sendo o $\mathrm{y}$, a idade de Joana ser o $x$, ao qual foi multiplicado por 3 entendendo-se que é o triplo, do qual o resultado seria 27 menos 6 . Onde na verdade a equação algébrica que corresponde corretamente ao exemplo (2) é $3 \cdot x-6=27$.

O critério de univocidade semântica terminal (II) concerne ao significado de cada unidade significante, ou seja, as unidades significantes do registro de partida precisam corresponder ao mesmo significado do registro de chegada. Observemos o exemplo 3:

(3) João foi a uma loja de roupas e comprou uma camiseta e uma bermuda, totalizando o valor de $R \$ 196,00$. A bermuda custou mais caro que a camiseta, a diferença foi de $R \$ 87,00$. Quanto custou cada peça que João comprou?

Convertendo o exemplo (3) no registro algébrico obtemos a equação $x+(x+87)=$ 196 , ao qual $x$ representa o valor da camiseta e $(x+87)$ o valor da bermuda. No entanto, o enunciado em linguagem natural traz a palavra diferença que semanticamente corresponde ao significado de subtração na matemática, mas na conversão ao registro algébrico a operação efetuada foi de adição ao invés da subtração, chegando à conclusão que não existe univocidade semântica terminal, haja vista que a unidade 
significante diferença está associada ao seu antônimo.

Para que existisse univocidade semântica terminal no enunciado (3) poderíamos realizar um tratamento:

(4) João foi a uma loja de roupas e comprou uma camiseta e uma bermuda, totalizando o valor de $R \$ 196,00$. A bermuda custou a mais que a camiseta $R \$ 87,00$. Quanto custou cada peça que João comprou?

Nesse caso a palavra mais está diretamente relacionada ao sinal da operação de adição (+) que está representado no registro algébrico. Dessa forma, "duas representações são congruentes quando há correspondência semântica entre suas unidades significantes, univocidade semântica terminal e mesma ordem possível de apreensão dessas unidades nas duas representações" [2] (p. 69). No entanto, pode acontecer de um, dois ou os três critérios não serem satisfeitos, então se tem uma não congruência entre duas representações, e esta pode ser em maior ou menor grau. A dificuldade na conversão de uma representação a outra varia dependendo do grau de não congruência entre elas.

Consideramos nesse estudo três graus de não congruência semântica [9]:

- Grau de não congruência semântica baixo: ao qual um dos critérios estabelecidos [2] não é satisfeito entre a representação de partida e a representação de chegada.

- Grau de não congruência semântica médio: dois dos critérios elencados [2] não são satisfeitos entre a representação de partida e a representação de chegada.

- Grau de não congruência semântica alto: nesse caso, nenhum dos critérios estabelecidos [2] são respeitados entre duas representações.

Evidenciaremos graus diferentes de não congruência semântica utilizando exemplos relacionados a função quadrática. A Figura 1 exemplifica o caso do grau de não congruência semântica baixo.

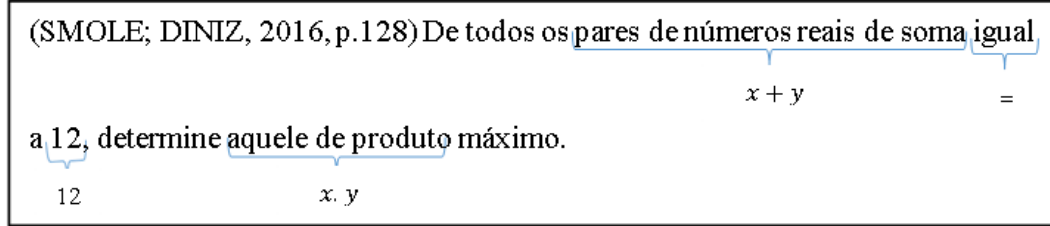

Figura 1: Exemplo de enunciado com grau de não congruência baixo [9] (p. 611).

Pela Figura 1 percebemos que a ordem dentro da organização das unidades significantes (III) é satisfeita, pois a representação algébrica segue a mesma ordem da 
representação em linguagem natural. Assim como o critério de univocidade semântica terminal (II) também é respeitado, pois as unidades significantes "soma" e "produto" estão representadas com o símbolo que elas significam na matemática (+) e (·), respectivamente.

Porém, a correspondência semântica dos elementos significantes (I) não acontece na conversão de uma representação a outra, já que os termos "pares de números reais de soma" possuem quatro signos - pois a preposição "de" não consideramos como unidade significante, já que ela apenas completa o sentido do enunciado - enquanto que na representação algébrica possui apenas três signos $(x+y)$. O que também pode ser notado no termo "aquele de produto", que possui dois signos contra três signos da representação algébrica $(x \cdot y)$. Portanto, da Figura 1 , existem dois critérios que foram satisfeitos e um que não foi correspondido.

No que concerne ao grau de não congruência médio, observemos o exemplo disposto na Figura 2.

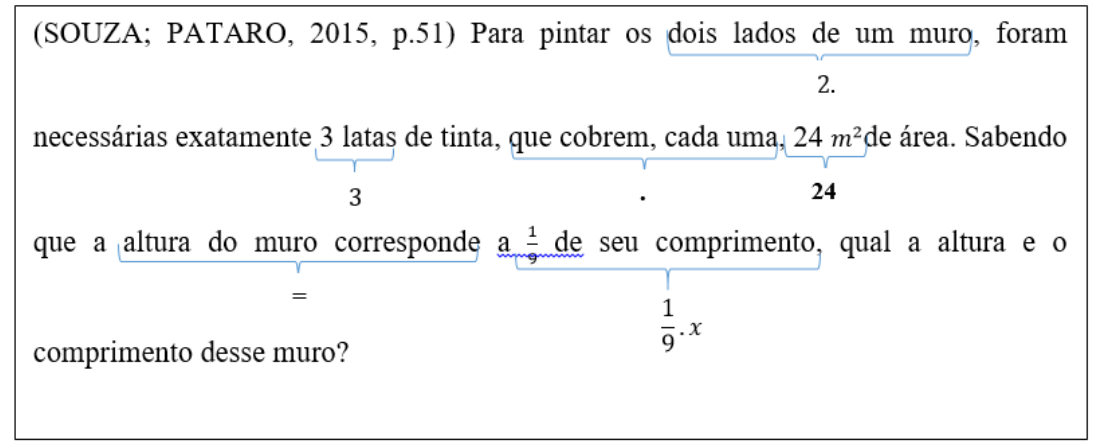

Figura 2. Exemplo de enunciado com grau de não congruência médio [8] (p.33)

Figura 2: Exemplo de enunciado com grau de não congruência médio [8] (p. 33).

Este exemplo na Figura 2, traz uma relação entre a altura e o comprimento de um muro, que de acordo com as informações, pode-se inferir que é de formato retangular. De início já constata-se que a ordem dentro da organização das unidades significantes (III) não acontece ao se converter a representação em linguagem natural para a representação algébrica que seria $72=2 \cdot x \cdot \frac{1}{9} \cdot x$, haja vista, que não segue a mesma ordem de leitura da esquerda para a direita.

Além disso, para chegar a área total do muro o estudante teria que converter o trecho "foram necessárias exatamente 3 latas de tinta, que cobrem, cada uma, $24 \mathrm{~m}^{2}$ de área" para $24 \cdot 3=72 \mathrm{~m}^{2}$, por isso a correspondência semântica dos elementos significantes (I) também não acontece, já que o termo "que cobrem, cada uma" que possuem quatro signos considerados representa apenas um signo, o sinal de multiplicação (·), ao ser efetuada a conversão.

No entanto, a univocidade semântica terminal (II) é satisfeita, pois não existem 
palavras com significados antônimos, como no caso da utilização de diferença com significado de adição, disposto no exemplo (3).

Para exemplificar um enunciado cujo grau de não congruência semântica é alto, ou seja, que os três critérios estabelecidos [2] não são correspondidos, verifiquemos o exemplo na Figura 3.

De todos os retângulos de perímetro igual a $60 \mathrm{~cm}$, determine o de área máxima.

$P$

60

Figura 3: Exemplo de enunciado com grau de não congruência alto [8] (p. 33).

Para encontrarmos a representação de chegada a partir desse enunciado (representação de partida), temos que recorrer a uma representação intermediária ou auxiliar para conseguir evidenciar unidades significantes que não estão presentes na representação em linguagem natural. Recorreremos, portanto, a representação auxiliar que corresponde a representação de um retângulo.

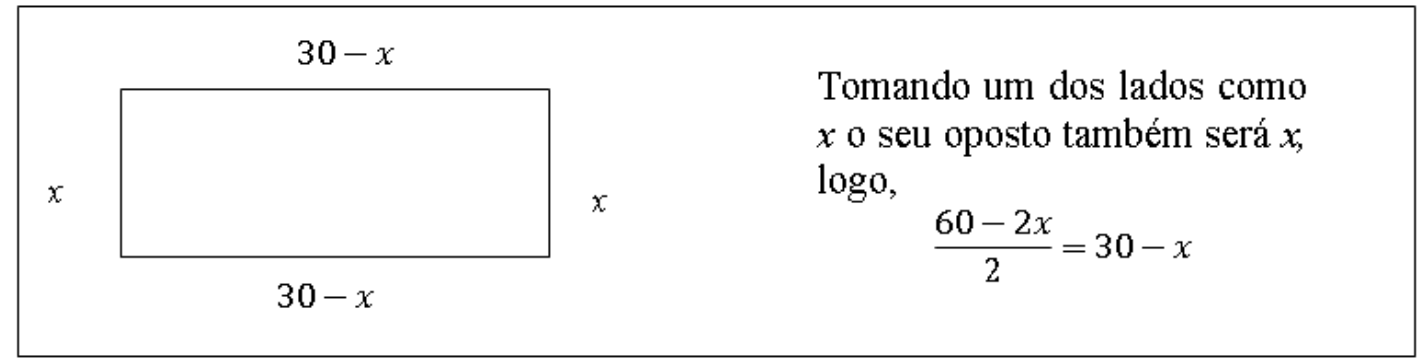

Figura 4: Representação auxiliar para resolução do exemplo da figura 3 [8] (p. 34).

Ao observamos a Figura 3 e a Figura 4 não encontraremos transparência de um registro a outro, pois em nenhum momento no registro em linguagem natural faz-se menção aos valores visuais da representação auxiliar, mas esta última, ainda não é a representação de chegada, tem apenas a função de ajudar na conversão. O registro de chegada para esse exemplo é $A(x)=x \cdot(30-x)$, mas para chegar a esse registro o estudante precisa saber como se calcula a área do retângulo que é dada pelo produto da base pela altura.

Assim, as unidades significantes essenciais para o registro de chegada não estavam presentes no registro de partida. Então, não houve correspondência semântica dos elementos significantes, univocidade semântica terminal e nem ordem dentro da organização das unidades significativas.

Portanto, o fenômeno da variação de congruência ou não congruência semântica é um dos causadores de dificuldades e erros na interpretação de enunciados em linguagem natural [3], exatamente porque a mente humana segue uma congruência semântica, e na maioria dos casos os enunciados revelam uma equivalência referencial, 
mas não uma congruência semântica, no entanto, pode ocorrer também o contrário, existir a congruência semântica e não ter a equivalência referencial [1].

Nesse sentido, a partir do estudo mais amplo realizado no mestrado em Educação Matemática e Tecnológica (Registro de Representação Semiótica e Função Quadrática: um olhar sobre o ensino e a abordagem no livro didático), selecionou-se para esse recorte a análise da variação de congruência e não congruência semântica e equivalência referencial nas atividades de conversão no sentido RLN para RA em função quadrática num livro didático de matemática para o $1^{\circ}$ ano (série) do ensino médio.

\section{DELINEAMENTO METODOLÓGICO}

A análise foi realizada no capítulo de função quadrática do livro didático (LD), versão do professor, aprovado pelo Plano Nacional do Livro Didático (PNLD) 2018 que permanece em vigência até 2020: volume 1 (referente a $1^{\text {a }}$ série do $E M$ ) da coleção MATEMÁTICA: CIÊNCIA E APLICAÇÕES de Gelson lezzi, Osvaldo Dolce, David Degenszajn, Roberto Périgo e Nilze de almeida. Publicado pela editora Saraiva, 9a edição no ano de 2016.

Este capítulo é composto por 158 itens em 67 questões, sendo contabilizado os exemplos, exercícios resolvidos, propostos e outras atividades que o livro didático propõe. Chamamos itens cada uma das solicitações das questões, exemplos, a, b, c etc). Vale salientar que as divisões expostas na Tabela I são identificados de acordo com subtítulos expostos no LD.

Tabela I: Distribuição de atividades no LD.

\begin{tabular}{|l|c|c|c|c|c|}
\hline & Exemplos & $\begin{array}{c}\text { Exercícios } \\
\text { resolvidos }\end{array}$ & Troque ideias & $\begin{array}{c}\text { Exercícios (para } \\
\text { responder) }\end{array}$ & Desafio \\
\hline Questões & 16 & 2 & 1 & 47 & 1 \\
\hline Itens & 16 & 4 & 3 & 134 & 1 \\
\hline
\end{tabular}

Para este texto, foram selecionadas as atividades que objetivavam a conversão RLN para RA. Pelos subtítulos da Tabela I traz-se a quantidade de itens analisados para a conversão supracitada, exposto na Tabela II.

Tabela II: Quantidade de itens para a conversão RLN $\rightarrow$ RA.

\begin{tabular}{|l|c|c|c|c|c|}
\hline & Exemplos & $\begin{array}{c}\text { Exercícios } \\
\text { resolvidos }\end{array}$ & Troque ideias & $\begin{array}{c}\text { Exercícios (para } \\
\text { responder) }\end{array}$ & Desafio \\
\hline Itens & 0 & 4 & 1 & 40 & 1 \\
\hline
\end{tabular}

As demais atividades envolvem outros tipos de conversões, como do registro algébrico para o registro gráfico, e a transformação de tratamento que acontece dentro do mesmo registro, a exemplo temos atividades que objetivavam encontrar as raízes ou vértice da função quadrática. Ainda, pontua-se que para a conversão $R L N \rightarrow R A$, a 
maior parte dos itens fazia-se necessário o uso de alguma representação auxiliar, seja ela, no próprio registro algébrico (RA), registro tabular (RT) ou registro figural (RF).

Para essa análise, procura-se responder aos questionamentos: Os três critérios correspondência semântica entre os elementos significantes, univocidade semântica terminal e ordem dentro da organização das unidades [2] são estabelecidas ou não na conversão entre as representações $\mathrm{RLN} \rightarrow \mathrm{RA}$ apresentadas no livro didático? $\mathrm{O}$ que se pode verificar quanto aos níveis de não congruência nas conversões entre as representações $\mathrm{RLN} \rightarrow \mathrm{RA}$ apresentadas pelo LD?

\section{ANÁLISE E DISCUSSÃo dOS RESULTADOS}

Foram analisados 46 itens sobre função quadrática cuja conversão é $R L N \rightarrow R A$, apresentados no livro didático. Desse total, as conversões que utilizam alguma manipulação/tratamento no RA antes de chegar ao registro final, que também é RA foram 33 itens. Os que usam o registro figural como representação auxiliar foram 2 itens. Apenas 1 usa o registro tabular e o tratamento em RA. E os demais, 10 itens, não referenciam a necessidade de nenhuma representação auxiliar ou tratamento. Mas, é possível perceber que grande parte das conversões $\mathrm{RLN} \rightarrow \mathrm{RA}$ são indiretas, isto é, necessitam de representações auxiliares.

Para as conversões do $\mathrm{RLN} \rightarrow \mathrm{RA}$ usando o tratamento em RA, tem-se os 4 itens dos exercícios resolvidos e 29 itens dos exercícios propostos. O Quadro 1 elucida se foram ou não conservados os critérios, em exercícios resolvidos, pontuados por Duval [2]: correspondência semântica entre os elementos significantes (A), univocidade semântica terminal (B) e ordem dentro da organização das unidades (C). Caso tenha sido conservado na questão será representado por (C), se não for conservado a representação será X (essa leitura serve para os demais quadros que analisam a conservação dos critérios).

O exercício resolvido 1, disposto no Quadro 1, possui três itens ( $\mathrm{a}, \mathrm{b}$ e c) que precisam ser convertidos da linguagem natural para algébrica, a fim de resolver a questão. No enunciado, além do registro em linguagem natural tem o registro algébrico de uma função, contudo, este registro não implicará na facilidade em resolver o exercício.

Pelo Quadro 1, o exercício resolvido 1 tem um grau de não congruência alto, em seus três itens:

\section{a) Não existem raízes reais}

Esse item corresponde a $\Delta<0$ em linguagem algébrica, assim a coordenação entre as unidades de sentido no RLN é raízes reais implicando em $\Delta$, no RA, e não existem implicando em $<0$ levando em consideração a associação entre o valor de 
delta e as raízes da função quadrática. Só que raízes reais têm duas unidades significantes para apenas uma, o $\Delta$. Logo, não existe correspondência semântica nas unidades significativas (A) e nem univocidade semântica terminal (B).

A ordem na organização das unidades significantes (C) em cada uma das representações também não é conservada. Como nenhum dos critérios foram satisfeitos, então o grau de não congruência é alto.

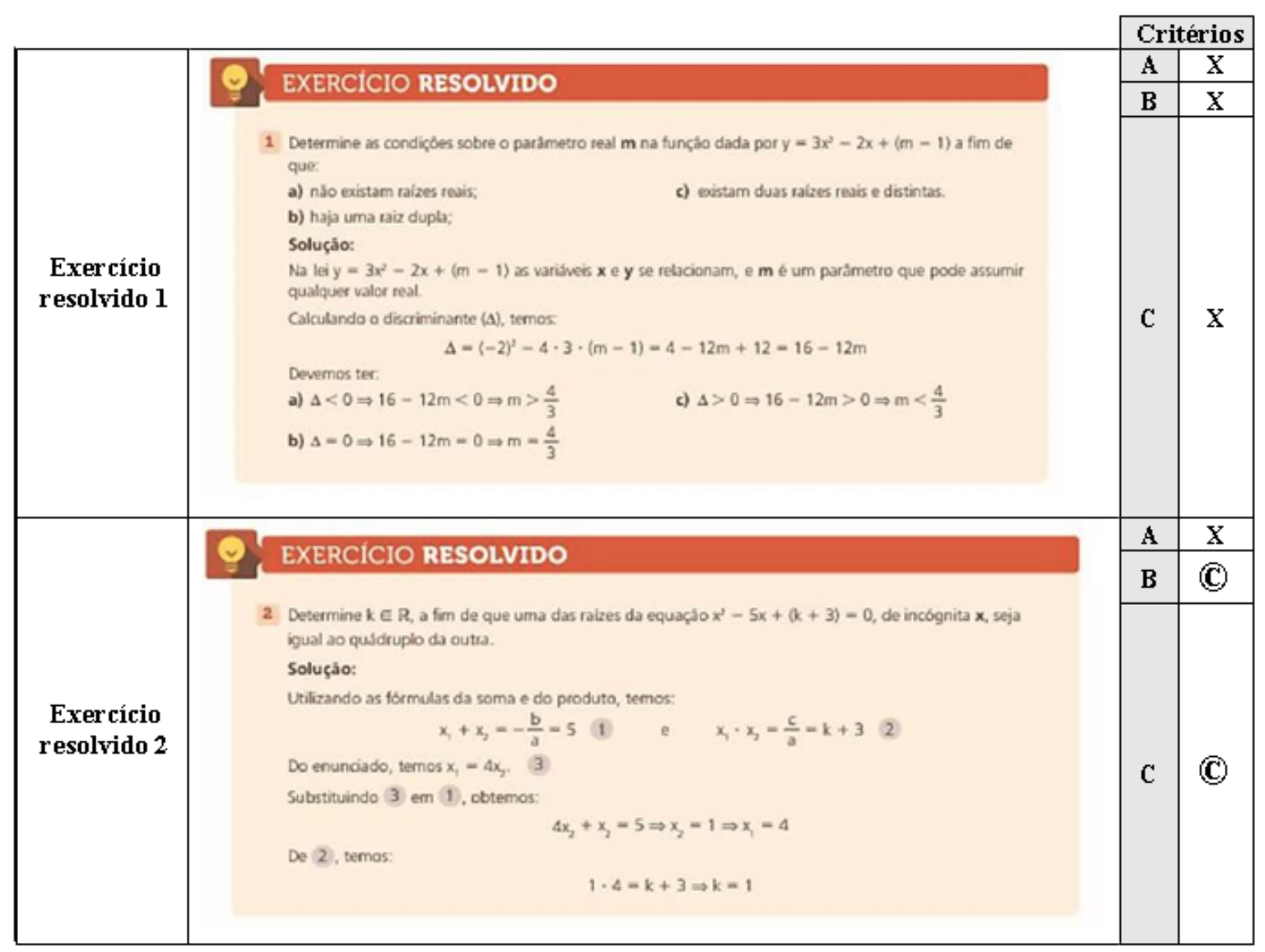

Quadro 1: Itens de conversão $\mathrm{RLN} \rightarrow \mathrm{RA}$ (usando o RA) com análise da conservação dos critérios de congruência.

\section{b) Haja uma raiz dupla}

A expressão algébrica correspondente ao registro em linguagem natural é $\Delta=0$. Porém o aluno pode escrever outra expressão: $x_{1}=x_{2}$, em que $x_{1}$ e $x_{2}$ sejam raízes da função quadrática, já que a unidade significante no RLN é uma raiz dupla. Os três critérios seguem o mesmo padrão do item anterior.

\section{c) Existem duas raízes reais e distintas}

A expressão que representa esse item é $\Delta>0$, todavia os estudantes poderiam escrever como $x_{1} \neq x_{2}$, já que são duas raízes e distintas. 
Se ao invés de existam duas raízes reais e distintas, estivesse escrito que o valor de $\Delta$ é positivo, o grau de não congruência semântica seria baixo, já que os critérios de univocidade semântica terminal (B) e ordem nas unidades significantes (C) seriam estabelecidos.

Por isso, existe um grau de não congruência alto nos três itens, pois nenhum critério é conservado. O que evidencia uma grande dificuldade nessa conversão, para os alunos.

Já no segundo exercício resolvido são conservadas a ordem das unidades significantes (C) e a univocidade semântica terminal (B). No entanto, a correspondência semântica das unidades significantes não é estabelecida, haja vista que existe mais de uma palavra em linguagem natural que ao ser convertida resulta em apenas um símbolo no registro algébrico (Figura 5).

2 Determine $k \in R$, a fim de que uma das raizes ja equaçăo $x^{2}-5 x+(k+3)-0$, de incógnita $x$, seja $\underbrace{\text { igual aoquádruplo, da }}_{=} \underbrace{\text { outra, }}_{4}$

Figura 5: A correspondência semântica no exercício resolvido 2, adaptado de [5] (p. 101).

Portanto, não há correspondência semântica, pois quádruplo (apenas uma palavra) refere-se a dois símbolos o 4 e o sinal de multiplicação (·).

A Tabela III exibe a classificação dos itens quanto aos critérios de congruência nas conversões $\mathrm{RLN} \rightarrow \mathrm{RA}$ com aspectos em RA nos enunciados dos exercícios.

Tabela III: Distribuição dos itens para as conversões RLN $\rightarrow$ RA com aspectos em RA.

\begin{tabular}{|c|c|c|c|c|}
\hline Congruente & $\begin{array}{c}\text { Baixo grau de não } \\
\text { congruência } \\
\text { semântica }\end{array}$ & $\begin{array}{c}\text { Médio grau de } \\
\text { não congruência } \\
\text { semântica }\end{array}$ & $\begin{array}{c}\text { Alto grau de não } \\
\text { congruência } \\
\text { semântica }\end{array}$ \\
\hline \multirow{2}{*}{ Itens } & $29(\mathrm{a}) ; 38(\mathrm{~b}) ; 48$ & $7 ; 10(\mathrm{~b}) ; 23 ; 29(\mathrm{~b}$ e & $10(\mathrm{a}$ e c); $14 ; 18$ & $12 ; 13 ; 15 ; 18(\mathrm{~b}) ;$ \\
& $(\mathrm{a})$. & $\mathrm{d}) ; 30(\mathrm{a}, \mathrm{b}$ e c);38 & $(\mathrm{a}) ; 29(\mathrm{c}) ; 31(\mathrm{a}$ e & $19 ; 20$. \\
\hline
\end{tabular}

No Quadro 2 selecionamos os enunciados que contemplam os três graus de não congruência e o que categorizamos como congruente para exemplificar a análise ${ }^{3}$, mas salientamos que é possível verificar os enunciados de todos os itens e as conservações dos critérios no quadro disposto na dissertação 4 ao qual foi realizado o recorte. Após o Quadro 2, são elucidados os critérios não conservados, em cada um dos 3 itens (Exercício 15 e 29) com graus de não congruência diferentes.

\footnotetext{
${ }^{3}$ Devido a quantidade de enunciados dos itens, selecionamos duas questões para exemplificar como foi realizada a análise. A análise dos itens completos estão na dissertação já enunciada.

${ }^{4}$ Quadro 16 (SILVA, 2020, p. 103-106).
} 


\begin{tabular}{|c|c|c|c|c|c|}
\hline \multirow{4}{*}{$\begin{array}{c}\text { Exercício } \\
15\end{array}$} & & \multirow{4}{*}{$\begin{array}{l}\text { Qual é o menor número inteiro } p \text { para o qual a } \\
\text { funçăo } f \text {, de } R \text { em } R \text {, dada por } f(x)=4 x^{2}+3 x+ \\
+(p+2) \text {, nâo admite raizes reais? }\end{array}$} & \multicolumn{3}{|c|}{ Critérios } \\
\hline & & & \multicolumn{2}{|c|}{$\mathrm{A}$} & $\mathrm{X}$ \\
\hline & & & \multicolumn{2}{|c|}{ B } & $\mathrm{X}$ \\
\hline & & & \multicolumn{2}{|c|}{$\mathrm{C}$} & $X$ \\
\hline \multirow{9}{*}{$\begin{array}{c}\text { Exercício } \\
29\end{array}$} & \multirow{9}{*}{29} & \multirow{5}{*}{$\begin{array}{l}\text { Uma bola, lançada verticalmente para cima, a partir } \\
\text { do solo, tem sua altura } \mathbf{h} \text { (em metros) expressa em } \\
\text { funçăo do tempo } t \text { (em segundos), decorrido aposs } \\
\text { o lançamento, pela lei: } \\
\qquad h(t)=40 t-5 t^{7}\end{array}$} & \multirow{3}{*}{ 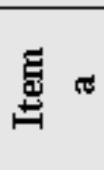 } & $\mathbf{A}$ & (c) \\
\hline & & & & B & (c) \\
\hline & & & & $\mathrm{C}$ & (c) \\
\hline & & & \multirow{3}{*}{ 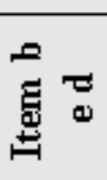 } & $\mathbf{A}$ & (c) \\
\hline & & & & B & (c) \\
\hline & & \multirow{4}{*}{$\begin{array}{l}\text { Determine: } \\
\text { a) a altura em que a bola se encontra } 1 \mathrm{~s} \text { após o } \\
\text { lançamento; } \\
\text { b) o(s) instante(s) em que a bola se encontra a } \\
75 \text { m do solo; } \\
\text { c) a altura máxima atingida pela bola: } \\
\text { d) o instante em que a bola retorna ao solo. }\end{array}$} & & $\mathrm{C}$ & $\mathrm{X}$ \\
\hline & & & \multirow{3}{*}{$\stackrel{\bullet}{\rightleftarrows}$} & $\mathrm{A}$ & $\mathrm{X}$ \\
\hline & & & & $\mathrm{B}$ & $\mathrm{X}$ \\
\hline & & & & $\mathrm{C}$ & (c) \\
\hline
\end{tabular}

Quadro 2: Análise da conservação de congruência em alguns exercícios com conversões $\mathrm{RLN} \rightarrow \mathrm{RA}$ que possuem aspectos em RA nos enunciados.

O item a do exercício 29, possui congruência semântica visto que os critérios de correspondência semântica das unidades significantes $(A)$, univocidade semântica terminal (B) e a ordem dentro dos elementos significantes (C) foram obedecidas.

O exercício 15 possui grau de não congruência semântica alto, haja vista que não conserva nenhum dos critérios estabelecidos por Duval [2]. No que concerne ao critério (C), apresenta ordem de organização entre os elementos significantes no RLN distintos dos elementos significantes no RA. Quanto ao critério de correspondência semântica dos elementos significantes (A), "não admite raízes reais" não faz correspondência um a um com $\Delta<0$; $E$ a univocidade semântica terminal (B) não é conservada porque "não admite raízes reais" pode admitir dois sentidos no RA ( $\Delta<0$ e $x_{1}$ e $\left.x_{2} \nexists \mathbb{R}\right)$.

O item c do exercício 29 foi classificado com grau médio de não congruência semântica em que não se conservam os critérios (A), pois, não existe uma correspondência biunívoca entre as unidades de sentido das duas representações convertidas e (B) houve mais de um sentido ao converter uma representação em outra. Apenas a ordem dentro da organização das unidades significantes (C) é conservada. Isto é, "altura máxima" que tem dois elementos significantes corresponde a apenas um, $y_{v}$ (a ordenada do vértice), mas nesse quesito a ordenada do vértice é $h$ e não $y$.

Os itens 29 itens $\mathbf{b}$ e $\mathbf{d}$ foram classificados com baixo grau de não congruência, por apresentarem ordem dentro da organização (C) diferentes entre os elementos significantes nos dois registros, por exemplo, o item d pede o instante em que a bola 
retorna ao solo, mas o instante faz referência a $t$ no RA, e ele vem após o $h$ que está relacionado à altura.

No Quadro 3 são apresentados a conservação dos critérios de congruência semântica nos dois itens em que a conversão é do $R L N \rightarrow R A$ usando o registro figural (RF). Eles possuem grau baixo de não congruência semântica, deixando de conservar apenas a ordem na organização das unidades significantes de cada uma das representações (C), já que todas essas unidades não estão presentes no RLN, parte delas estão no registro auxiliar (RF) que possui dimensão 2D, diferente do RA que possui dimensão 1D. Como as dimensões são diferentes, normalmente, a ordem não é estabelecida.

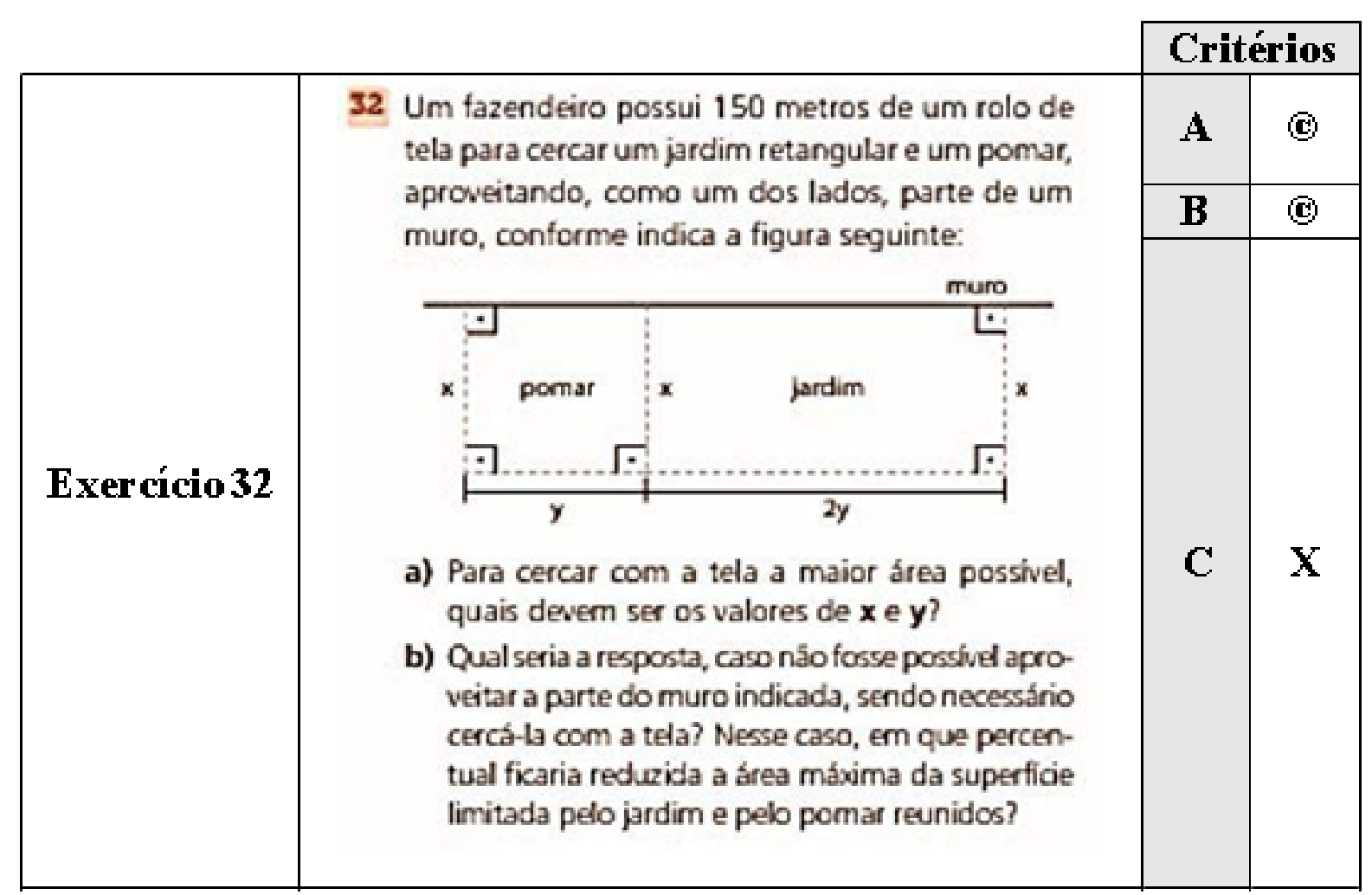

Quadro 3: Análise da conservação dos critérios de congruência semântica no exercício com conversão $\mathrm{RLN} \rightarrow \mathrm{RA}$ usando o registro figural (RF).

O Quadro 4 evidencia a classificação da conservação dos critérios de congruência nas conversões do $\mathrm{RLN} \rightarrow \mathrm{RA}$, sem a interferência de nenhum registro auxiliar.

\begin{tabular}{|c|c|c|c|}
\hline & & \multicolumn{2}{|c|}{ Critérios } \\
\hline \multirow{3}{*}{ Exercício8 } & \multirow{3}{*}{$\begin{array}{l}\text { B. Em um retångulo, a medida de um dos lados exceda } \\
\text { a medida do outro em } 4 \mathrm{~cm} \text {. Sabendo que a área } \\
\text { desseretângulo } 621 \mathrm{~cm}^{2} \text {, determineseu perimetro. }\end{array}$} & $\mathbf{A}$ & $\mathrm{X}$ \\
\hline & & B & (c) \\
\hline & & C & $\mathbf{X}$ \\
\hline
\end{tabular}




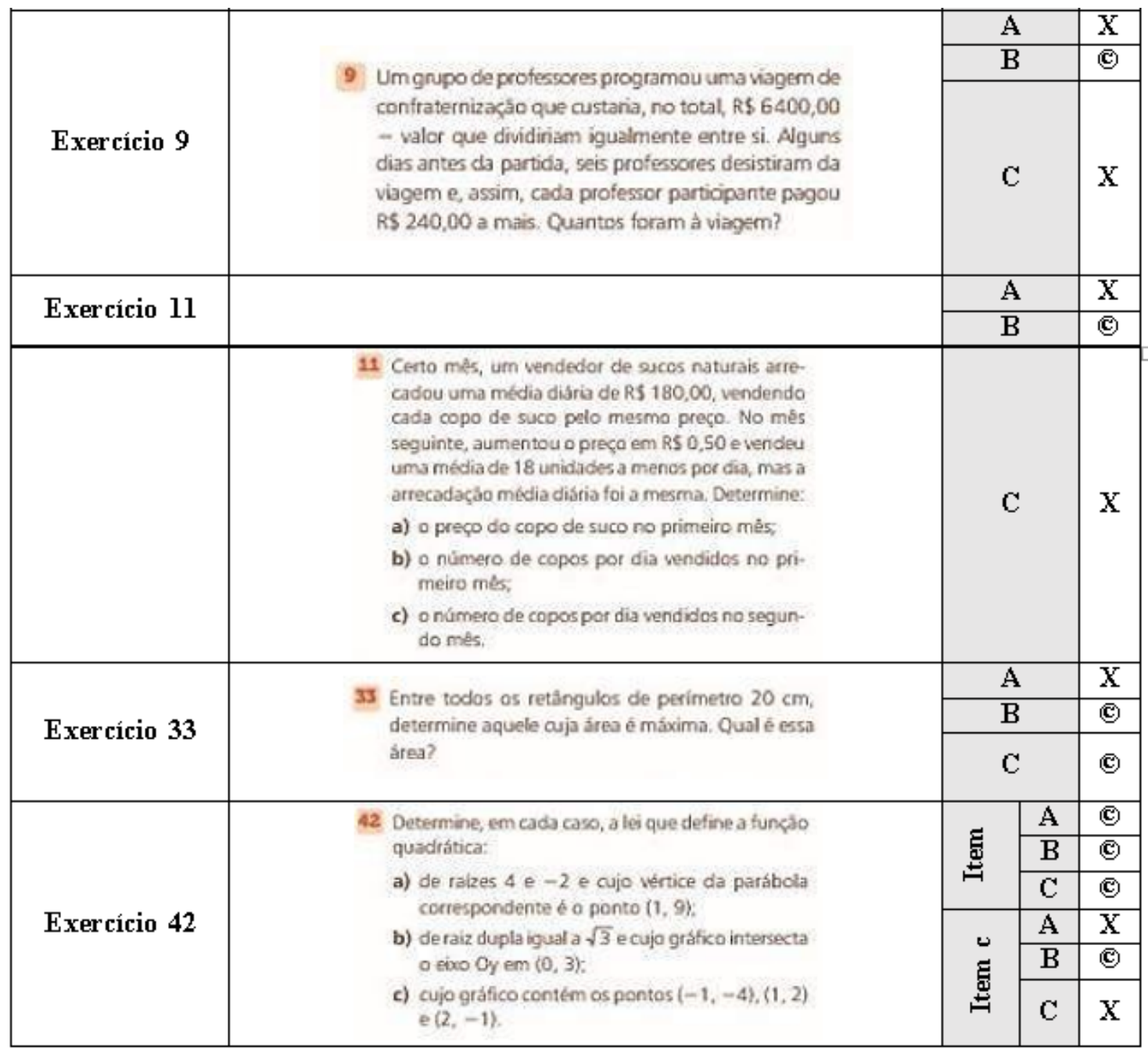

Quadro 4: Análise da conservação dos critérios de congruência semântica nos exercícios com conversão RLN $\rightarrow$ RA.

Pelo Quadro 4, os itens 8, 9 e 11 (a, b, c) se caracterizam como grau médio de não congruência semântica, por não conservarem dois dos três critérios: $(A)$ e (C). De (C) a ordem dos elementos significantes entre as representações convertidas não são as mesmas. $E$ de (A) não existe correspondência biunívoca entre as unidades de sentido de cada uma das representações, o que pode ser observado na conversão ( $R L N \rightarrow R A)$ das unidades significantes de cada uma delas nos tópicos abaixo:

- 8 - "a medida de um dos lados excede a medida do outro em $4 \mathrm{~cm}$ " "área desse retângulo é $621 \mathrm{~cm}^{2} " \rightarrow x \cdot(x+4)=621$.

- 9 - "R\$6400,00 - valor que dividiriam igualmente entre si" "seis professores desistiram da viagem" "cada professor pagou $\mathrm{R} \$ 240,00$ a mais" $\rightarrow \frac{6400}{n}$ e $\frac{6400}{n-6}+240$.

- 11 itens a, b e c - "arrecadou uma média diária de $\mathrm{R} \$ 180$, vendendo cada copo de suco pelo mesmo preço" $\rightarrow n \cdot p=180$; "aumentou o preço em $\mathrm{R} \$ 0,50 \mathrm{e}$ 
vendeu uma média de 18 unidades a menos por dia, mas a arrecadação média diária foi a mesma" $\rightarrow(p+0,50) \cdot(n-18)=180$.

Nesse caso, o enunciado se encarrega por denotar todas as unidades significantes, e os itens são resolvidos com base nas informações do enunciado. O que, normalmente, é encontrado como resolução no item a, irá auxiliar na resolução do item b, por exemplo. Ainda assim, o grau de não congruência semântica chega a ser menor.

O item que se revela com baixo grau de não congruência semântica é o 33. Apenas, não conserva o critério $(A)$ já que as unidades de sentido de uma representação não se relacionam biunivocamente, pois "retângulos de perímetro $20 \mathrm{~cm}$ " com três elementos significantes, corresponde a $2 x+2 y=20$, no RA com mais que três.

O item 42 (c), não conserva os critérios (A) já que as unidades de sentido de uma representação não se relacionam biunivocamente e (C) pois as ordens apresentadas nos pares ordenados é $(x, f(x))$, e na lei de formação, esses elementos são posicionados, primeiro $f(x)$ e depois o $x$, se tomarmos $f(x)=a x^{2}+b x+c$. Quanto ao critério (A) "os pontos $(1,4),(1,2)$ e $(2,1)$ " não estão presentes no registro final, logo não há correspondência semântica.

Quanto aos itens a e b do exercício 42 que são categorizados como congruentes, pois conservam os três critérios de congruência, destaca-se que o critério (A) foi conservado devido ao uso da representação algébrica na forma fatorada, que evidencia as raízes.

O Quadro 5 concerne a variação de congruência e não congruência semântica no desafio, a análise acentua que se trata de um grau de não congruência médio, pois, não conserva dois dos critérios estabelecidos por Duval [2].

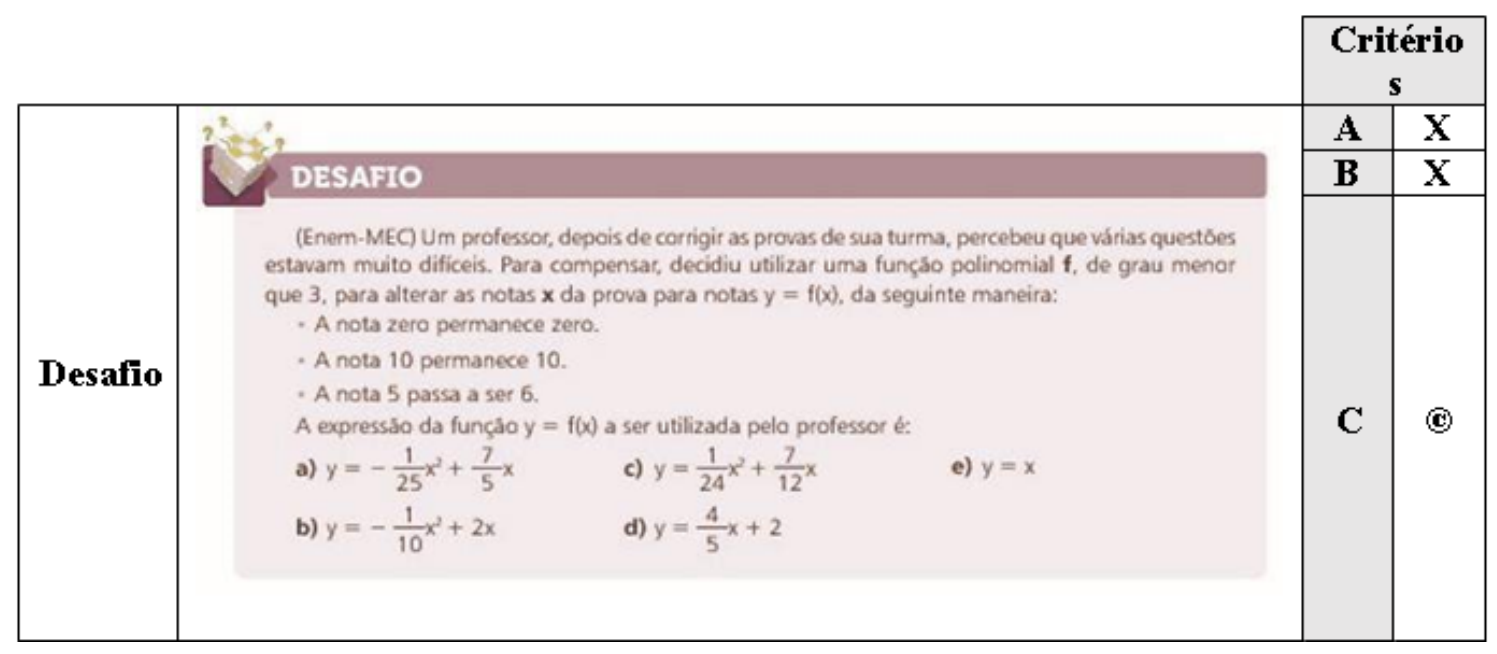

Quadro 5: Desafio com análise da conservação dos critérios de congruência.

O critério da univocidade semântica terminal (B) não é conservado, pois "função 
polinomial f, de grau menor que 3" pode ser compreendida de duas formas no registro em linguagem natural: $f(x)=a x+b$ ou $f(x)=a x^{2}+b x+c$, ambas leis de formação possuem grau menor que 3 .

A correspondência semântica terminal (A) não é conservada, pois, a cada termo significante do registro de partida, não é correspondido somente um no registro de chegada. E a ordem na organização das unidades significantes (C) é estabelecida, haja vista, que se segue em ambos os registros, de chegada e de partida.

A única questão que usa o registro tabular e o tratamento no RA, dentro da conversão do $\mathrm{RLN} \rightarrow \mathrm{RA}$ está apresentada na atividade troque ideias, que embora seja composta por três itens (Figura 6), apenas um enfatiza a conversão que abordamos nesse texto.

\section{TROOVE TDEIAS}

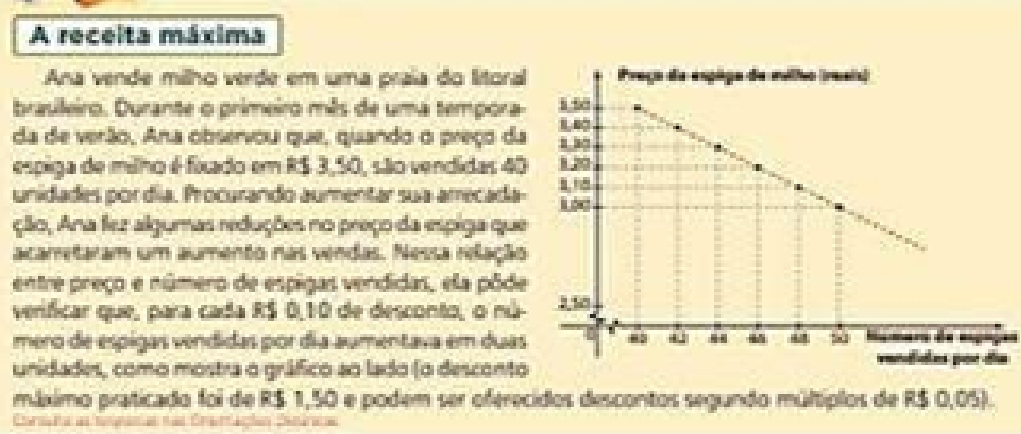

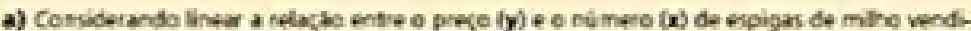

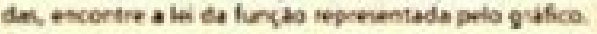

b) Copre no caderno e complete a tabela segaste. que relaciesa o prego da espipa de mine.

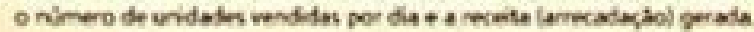

\begin{tabular}{|c|c|c|}
\hline $\begin{array}{l}\text { Prese da espigs } \\
\text { (NS) }\end{array}$ & $\begin{array}{l}\text { Nimero de } \\
\text { expigus wendidas } \\
\text { por dia }\end{array}$ & $\begin{array}{c}\text { Receits dubria } \\
\text { (Dis) }\end{array}$ \\
\hline 3.50 & & \\
\hline 3.40 & & \\
\hline 3,30 & & \\
\hline 3.00 & & \\
\hline 2.90 & & \\
\hline 2,80 & & \\
\hline 2,50 & & \\
\hline
\end{tabular}

C) No analisy a tabela, Ana fecu interessada em saber qual e prege a ser cobrode pela espiga que propordonaria a maior receita posivel, eto t, a roceisa mivena. Use seus conhecimentos pura reselver esse problema. No find, voct deve'b determinar:

i) o prego a ser cobrado pela unidude de espina:

in a quartidabe de woign vendidas por ese precpo

iii) a recehta gerads resias condiçbes.

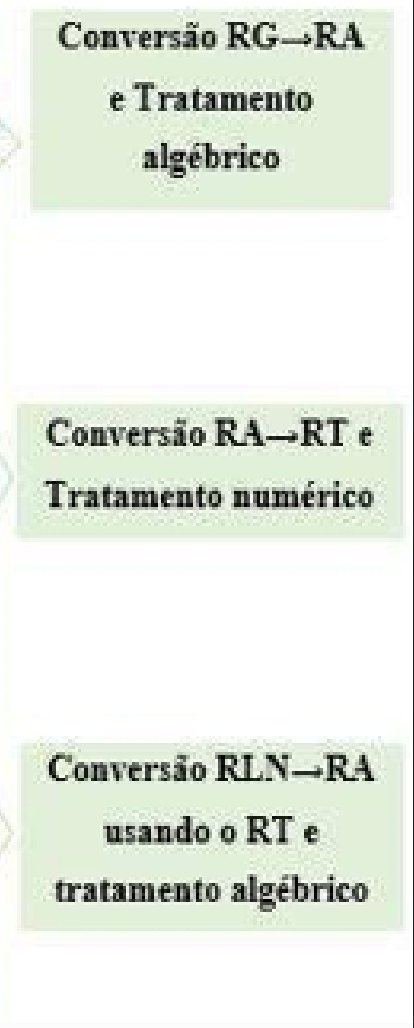

Figura 6: Transformação de conversão na seção troque ideias, adaptado de [5] (p. 105-106). 
Sendo assim, é analisada conservação dos critérios estabelecidos [2] apenas no item c desta atividade (Quadro 6).

\begin{tabular}{|c|c|c|c|}
\hline & & & \\
\hline \multirow[b]{3}{*}{ Item $\mathbf{c}$} & \multirow{3}{*}{ 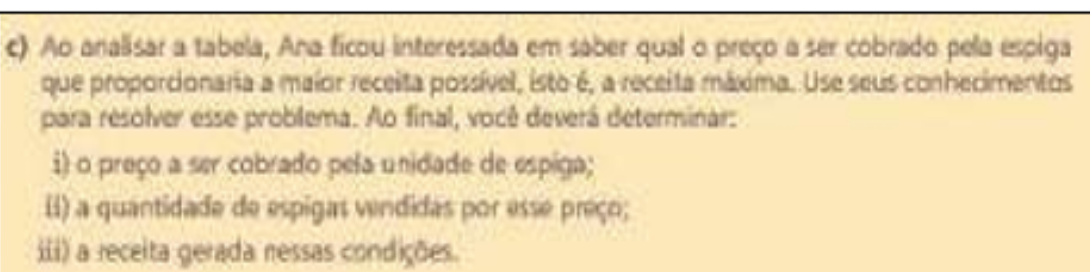 } & $\mathbf{A}$ & $\mathrm{X}$ \\
\hline & & B & (C) \\
\hline & & $\mathrm{C}$ & $\mathrm{X}$ \\
\hline
\end{tabular}

Quadro 6: Item c do troque ideias com análise de conservação dos critérios de congruência.

Pelo Quadro 6, o item c também se estabelece um grau de não congruência médio, pois a univocidade semântica terminal (B) é conservada, mas a ordem na organização das unidades significantes $(C)$ e a correspondência semântica (A) não são estabelecidas. Ao converter os enunciados, sabendo que Ana quer descobrir a receita máxima, temos:

i. O preço a ser cobrado pela unidade de espiga $\rightarrow \frac{y_{v}}{x_{v}}$;

ii. A quantidade de espigas vendidas por esse preço $\rightarrow x_{v}$;

iii. A receita gerada nessas condições $\rightarrow y_{v}$.

Comparando um registro a outro, as unidades significantes de um, não estão associados a apenas uma unidade significantes do outro, por isso não é conservada a correspondência semântica (A), embora existe os itens ii e iii possibilitam maior correspondência que o item i, já que nesse o RA corresponde a uma razão.

No critério $\mathrm{C}$, a ordem que se encontra no enunciado (registro de partida), não é a mesma no registro de chegada. Pois, são realizados alguns procedimentos: escrever a lei de formação da função quadrática que permitirá encontrar a receita máxima, daí encontra-se o ii., o iii. e por fim o i.

De maneira geral, as conversões do RLN para o RA, enquadram-se em baixo e médio grau de não congruência semântica, principalmente, os que estão dispostos nos exercícios propostos.

\section{CONSIDERAÇÕES FINAIS}

A análise do fenômeno de congruência semântica e equivalência referencial em 46 itens de atividades apresentadas em um livro didático da $1^{\mathrm{a}}$ série do ensino médio sobre função quadrática, mas especificamente, com foco nas conversões no sentido 
registro em linguagem natural $(R L N)$ para o registro algébrico (RA), apontam maior índice de atividades com baixo e médio grau de não congruência semântica. Apenas nove itens foram classificados com grau alto de não congruência semântica, e outros 5 itens foram classificados como congruentes.

Quanto mais alto o grau de não congruência, maior o esforço cognitivo do estudante para a interpretação e resolução do problema. Quanto mais transparente for a conversão, menor a dificuldade e maior índice de congruência semântica entre as representações convertidas. Por isso é importante, ao utilizar o livro didático como apoio para o seu planejamento e ensino, que o professor analise as atividades que irá propor aos seus alunos, variando as questões quanto ao esforço cognitivo a ser disposto na resolução, aprimorando assim a aprendizagem.

Salientamos que esta análise baseou-se apenas nos critérios enfatizados por Duval [2], mas que podem ser aprimorados se analisados com mais detalhes, sob o olhar minucioso de cada unidade significativa e elementos próprios do conceito de função. Para isso deixamos como proposta para estudos futuros.

\section{REFERÊNCIAS}

[1] R. Duval, Registros de representação semiótica e funcionamento cognitivo do pensamento, 1993. Tradução: M.T. Moretti. Revista eletrônica de Educação Matemática, Florianópolis, v. 07, n. 2, p. 266-297, 2012. [CrossRef] [Google Scholar]

[2] R. Duval. Semiósis e pensamento humano: registro semiótico e aprendizagens intelectuais (Sémiosis et Pensée Humaine: Registres Sémiotiques et Apprentissages Intellectuels). Tradução de L. F. Levy e M.R. A. da Silveira. São Paulo: Editora Livraria da Física, fascículo I, 2009.

[3] R. Duval, Ver e ensinar a matemática de outra forma: Entrar no modo matemático de pensar: os registros de representações semióticas. In: T.M.M. Campos (org). Tradução: M.A. Dias. São Paulo: PROEM, 2011.

[4] C. R. Flores; M. T. Moretti, O funcionamento cognitivo e semiótico das representações gráficas: Ponto de análise para aprendizagem Matemática". REUNIÃO ANUAL DA ANPED, GT19: EDUCAÇÃO MATEMÁTICA, 28., 2005, Caxambu. Anais... Caxambu: ANPED. p.1-13. Disponível em: <http://www.ufrrj.br/emanped/paginas/conteudo_producoes/docs_28/funcionamento. pdf $>$. Acesso em: $11 \mathrm{dez} .2019$.

[5] G. Iezzi; O. Dolce; D. Degenszajn; R. Périgo; N. Almeida, Matemática: Ciência e Aplicações. São Paulo: Saraiva, v.1, 9a ed., 2016.

[6] E. H. Lourenço; P. C. Oliveira, Congruência semântica e equivalência referencial em problemas envolvendo equações de $1^{\circ}{ }^{\circ}$ grau. Educação Matemática Pesquisa, São Paulo, v. 20, n.1, p. 084-109, 2018.

[7] K. S. Smole; M. I. Diniz, Matemática para compreender o mundo. São Paulo: Saraiva, 2016.

[8] A. S. Silva, Registros de representação semiótica e função quadrática: um olhar sobre o ensino e a abordagem no livro didático, dissertação de mestrado, Mestrado em Educação Matemática e Tecnológica, Universidade Federal de Pernambuco, 2020.

[9] A. S. Silva; R. A. M. Teles, Convergências entre a abordagem do livro didático e o ensino de função quadrática: um olhar sob o ponto de vista dos registros de representação semiótica. Educação Matemática Pesquisa. São Paulo, v. 22, n.2, p. 604-634, 2020. [CrossRef] [Google Scholar] 


\section{BREVE BIOGRAFIA}

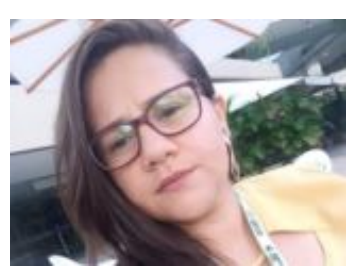

Andreza Santana da Silva (D) https://orcid.org/0000-0001-9675-3557

Doutoranda em Educação Matemática e Tecnológica pela Universidade Federal de Pernambuco, com início em 2020. Professora substituta de matemática no Colégio de Aplicação - UFPE. Integrante dos grupos de pesquisa SEMEAR, GEPeDiMA e Pró-grandezas.

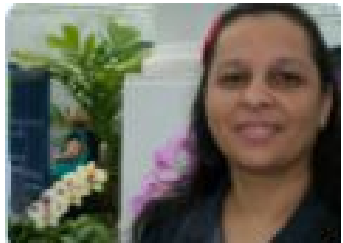

Rosinalda Aurora de Melo Teles (D) https://orcid.org/ 0000-0002-7289-3501

Doutora em Educação pela Universidade Federal de Pernambuco. Professora do Programa de Pós-Graduação em Educação Matemática e Tecnológica (EDUMATEC) da Universidade Federal de Pernambuco. 